\title{
Multiple biomarker strategy for improved diagnosis of acute heart failure in older patients presenting to the emergency department
}

European Heart Journal: Acute Cardiovascular Care 2015, Vol. 4(2) 137-147

(C) The European Society of Cardiology 2014

Reprints and permissions: sagepub.co.uk/journalsPermissions.nav DOI: 10.1 177/20488726/454|904 acc.sagepub.com

(SSAGE

\author{
Philipp Bahrmann 1*, Anke Bahrmann 2*, Benjamin Hofner ${ }^{3}$, \\ Michael Christ ${ }^{4}$, Stephan Achenbach ${ }^{2}$, Cornel Christian Sieber ${ }^{1}$ \\ and Thomas Bertsch ${ }^{5}$
}

\begin{abstract}
Aim: Biomarkers can help to identity acute heart failure (AHF) as the cause of symptoms in patients presenting to the emergency department (ED). Older patients may prove a diagnostic challenge due to co-morbidities. Therefore we prospectively investigated the diagnostic performance of $\mathrm{N}$-terminal pro-B-type natriuretic peptide (NT-proBNP) alone or in combination with other biomarkers for AHF upon admission at the ED.

Methods: 302 non-surgical patients aged $\geq 70$ years were consecutively enrolled upon admission to the ED. In addition to NT-proBNP, mid-regional pro-adrenomedullin (MR-proADM), mid-regional pro-atrial natriuretic peptide (MRproANP), C-terminal pro-endothelin-I (CT-proET-I) and ultra-sensitive C-terminal pro-vasopressin (Copeptin-us) were measured at admission. Two cardiologists independently adjudicated the final diagnosis of AHF after reviewing all available baseline data excluding the biomarkers. We assessed changes in $\mathrm{C}$-index, integrated discrimination improvement (IDI), and net reclassification improvement (NRI) for the multimarker approach.

Results: AHF was diagnosed in 120 (40\%) patients (age $81 \pm 6$ years, 64 men, 56 women). Adding MR-ADM to NT-proBNP levels improved C-index (0.84 versus $0.81 ; p=0.045)$, and yielded IDI (3.3\%; $p=0.002)$, NRI (I7\%, $p<0.00 \mathrm{I})$ and continuous NRI $(33.3 \% ; p=0.002)$. Adding CT-proET-I to NT-proBNP levels improved $C$ index ( 0.86 versus $0.8 I, p=0.03 I)$, and yielded robust IDI $(12.4 \% ; p<0.00 I), N R I(3 I .3 \%, p<0.00 I)$ and continuous NRI $(69.9 \% ; p<0.001)$. No other dual or triple biomarker combination showed a significant improvement of both C-index and IDI.

Conclusion: In older patients presenting to the ED, the addition of CT-proET-I or MR-proADM to NT-proBNP improves diagnostic accuracy of AHF. Both dual biomarker approaches offer significant risk reclassification improvement over NT-proBNP.
\end{abstract}

\section{Keywords}

adrenomedullin, aged, diagnosis, endothelin, heart failure, natriuretic peptides

Date received: 25 March 20I4; accepted: II June 2014

\footnotetext{
Institute for Biomedicine of Aging, Friedrich-Alexander-University, Nuremberg, Germany

2Department of Cardiology, Friedrich-Alexander-University, Erlangen,

Germany

${ }^{3}$ Department of Medical Informatics, Biometry and Epidemiology,

Friedrich-Alexander-University, Erlangen, Germany

${ }^{4}$ Department of Emergency and Critical Care Medicine, Paracelsus

Medical University, Nuremberg, Germany
}

\footnotetext{
${ }^{5}$ Institute for Clinical Chemistry, Laboratory Medicine and Transfusion Medicine, Paracelsus Medical University, Nuremberg, Germany *Both authors contributed equally to this paper.

Corresponding author:

Philipp Bahrmann, Institute for Biomedicine of Aging, FriedrichAlexander-University, Kobergerstr. 60, D-90408 Nuremberg, Germany. Email: philipp.bahrmann@gmail.com
} 


\section{Introduction}

Patients $>70$ years old constitute an increasing proportion of patients who presenting to the emergency department (ED) for diagnosis of acute heart failure (AHF). The diagnosis of AHF is challenging in older patients because symptoms and signs may be atypical and can be simulated or disguised by comorbid conditions such as chronic obstructive pulmonary disease (COPD), obesity, or chronic venous insufficiency. Moreover, cognitive impairment and hardness of hearing impairs diagnostic work-up. ${ }^{1,2}$

Management of AHF in older patients is associated with diagnostic challenges not adequately addressed in current guidelines. $^{3-5}$ For patients presenting to the ED with acute onset of symptoms, early echocardiography is recommended for suspicion of AHF. Because symptoms or signs of AHF are ambiguous, many older patients with suspected HF who undergo routine echocardiography are not correctly identified. ${ }^{6}$ In contrast to heart failure (HF) with reduced ejection fraction (HFrEF) the diagnosis of $\mathrm{HF}$ in patients with preserved ejection fraction (HFpEF) is challenging. Potential non-cardiac causes of the older patient's symptoms (such as anemia or COPD) must first be discounted. ${ }^{4}$ Echocardiography also does not consistently provide definitive results in the acute care setting due to the difficulties of imaging, particular in the elderly. ${ }^{2}$ The diagnosis of AHF ideally requires further accurate, accessible, cost-effective and acceptable evidence of cardiac dysfunction such as measurements of circulating levels of natriuretic peptides. Of note, the diagnostic threshold for the $\mathrm{N}$-terminal part of the precursor of B-type natriuretic peptide (NT-proBNP) increases not only with age - for example due to impaired renal function - but also differs for patients presenting with acute onset or worsening of symptoms at ED presentation and those who present with a more gradual onset of symptoms. ${ }^{4,7,8}$ To overcome the limitations of NT-proBNP in the diagnosis of AHF, the use of additional innovative biomarkers may be helpful to improve diagnostic discrimination in older patients presenting to the ED. ${ }^{2}$

Adrenomedullin (ADM) is a potent vasodilator peptide that is secreted predominantly from vascular endothelial cells. Previous studies have suggested that plasma ADM levels are increased in HF and after myocardial infarction, and are associated with increased risk. ${ }^{9}$ Atrial natriuretic peptide (ANP) is a sister peptide to the B-type natriuretic peptide and is diagnostically equivalent to NT-proBNP in HF. Endothelin-1 (ET-1) is a potent vasoconstrictor with elevated plasma levels in HF. Arginine vasopressin (AVP) levels are also known to be elevated in HF and its actions in promoting salt and water retention and as a vasoconstrictor are well documented. ${ }^{10}$

Several novel immunoassays for these biomarkers have been developed for the detection of the stable prohormone fragment as a 'mirror' of natural hormone release. ${ }^{11,12} \mathrm{New}$ immunoassays can be directed at stable prohormones such as mid regional ADM (MR-proADM), mid regional ANP (MR-proANP), C-terminal pro-Endothelin-1 (CT-proET-1) or ultra-sensitive C-terminal proAVP (Copeptin-us) that are stoichometrically related to synthesis of the biologically active unstable fragment. ${ }^{13}$

We investigated whether a multiple biomarker strategy may improve accuracy to diagnose AHF in consecutive older patients presenting to the emergency department. The objective of our study was to assess the value of a multimarker strategy by discrimination and reclassification measures for MR-proADM, MR-proANP, CT-proET-1, and Copeptin-us compared to NT-proBNP alone for the diagnosis of AHF. To assess the incremental value of a multimarker strategy for the diagnosis of AHF, we used NT-proBNP as the base clinical model and calculated: 1) changes in the $\mathrm{C}$ index; 2) the integrated discrimination improvement (IDI), which quantifies improvement in diagnostic separation; and 3) the net reclassification improvement (NRI) and continuous NRI, clinically oriented measures of reclassification that considers any correct upwards movement of events (in the new vs. the old model) and any downwards movement of non-events as model improvement, and vice versa. ${ }^{14,15}$

\section{Methods}

\section{Study design and population}

From 18 January 2011 to 5 September 2011, we measured NT-proBNP, MR-proADM, MR-proANP, CT-proET-1 and Copeptin-us as part of the routine blood sampling protocol upon admission of all consecutive non-trauma patients aged $\geq 70$ years who were admitted to the emergency department (ED) of a large tertiary care center in Nuremberg, Germany. Exclusion criteria were acute ST-elevation myocardial infarction, planned elective coronary revascularization, hospitalization for unstable angina within the preceding 2 months, coronary-artery bypass grafting or percutaneous transluminal angioplasty within the preceding 3 months. Patients were also excluded if they had renal failure requiring dialysis, trauma with suspected myocardial contusion, life expectancy $<6$ months, or if they did not consent to providing a blood sample for use by the research team. The study conformed to the principles outlined in the Declaration of Helsinki and was conducted after approval by the Ethics Committee of the Friedrich-AlexanderUniversity. All patients or their guardians provided written informed consent.

\section{Clinical characteristics}

All patients underwent a standard clinical workup, including patient history, physical examination, standard 12-lead electrocardiography (ECG), noninvasive blood pressure measurement, and laboratory analyses. The New York Heart 
Association (NYHA) classification, Barthel index and Charlson co-morbidity index were assessed in all patients. ${ }^{16-18}$ Bedside echocardiography (Vivid S6 cardiovascular ultrasound system, GE Healthcare, Madison, USA) was performed on all patients by an attending cardiologist at the ED. Left ventricular ejection fraction (LVEF) was calculated by Simpson's rule and wall motion severity index using the American Society of Echocardiography 16 segment model. ${ }^{19}$ Assessment of left ventricular systolic function allowed sub-classification of preserved (LVEF $\geq 55 \%$ ), subnormal $(\mathrm{LVEF}<45 \%)$ or borderline $(55 \%>\mathrm{LVEF} \geq 45 \%)$ LVEF function. Doppler and tissue-Doppler measurements of the longitudinal function of the heart were used to determine left ventricular diastolic dysfunction (LVDD). E/e' measurements were recorded at both the septum and lateral wall. ${ }^{20}$ All other diagnostic and therapeutic procedures were chosen at the discretion of the attending physicians at the ED and other departments.

\section{Adjudicated final diagnosis}

To determine the final diagnosis for each patient, two cardiologists ( $\mathrm{AB}$ and $\mathrm{PB}$ ) independently reviewed all available medical records of the index hospital stay, including the clinical history findings from the physical examination, results of laboratory tests (excluding NT-proBNP, MR-proADM, MR-proANP, CT-proET-1 and Copeptin-us assays), radiographic studies, ECG, and echocardiography. AHF was diagnosed when typical signs and symptoms in combination with objective evidence of an abnormality of cardiac structure or function at rest based on the definition of the ESC guideline was present. ${ }^{4}$ In patients with the adjudicated final diagnosis of AHF, HFpEF was diagnosed in accordance with ESC guideline using echocardiographic and NT-proBNP measurements. Patients whose LVEF was $>50 \%$ with $\mathrm{E} / \mathrm{e}^{\prime}>15$, or those with am equivocal $\mathrm{E} / \mathrm{e}^{\prime 8-15}$ but NT-proBNP $>220 \mathrm{pg} / \mathrm{mL}$ were diagnosed as having HFpEF. $^{20}$ Disagreement regarding final classification occurred in $4 \%$ of the cases and was resolved by consensus involving a third cardiologist (MC).

\section{Laboratory analysis}

Routine laboratory parameters, including high-sensitive cardiac troponin $\mathrm{T}$ (hs-cTnT), C-reactive protein, creatinine and $\mathrm{N}$-terminal pro-brain natriuretic peptide (NT-proBNP) were measured immediately after blood withdrawal by standardized methods. Additionally, potassium EDTA plasma samples for MR-proADM, MR-proANP, CT-proET-1, Copeptin-us and Cystatin $C$ measurement were collected at the time of the patient's admission to the ED, centrifuged and frozen at $-80^{\circ} \mathrm{C}$ until they were analyzed in a blinded fashion in one batch at the core laboratory of the Nuremberg Hospital during a secondary laboratory assessment. Laboratory analyses were done without knowledge of clinical presentation.
Cardiac troponin $\mathrm{T}$ was measured using the hs-cTnT assay (Roche Diagnostics, Mannheim, Germany) in all patients with electrochemiluminescence immunoassay (ELICA) technology on a cobas e411 system (Roche Diagnostics, Mannheim, Germany). The limit of blank was $0.003 \mathrm{ng} / \mathrm{mL}$ and the limit of detection $0.005 \mathrm{ng} / \mathrm{mL}$. The 99th percentile cut-off was $0.014 \mathrm{ng} / \mathrm{mL}$, which was used as the diagnostic cut-off. The coefficient of variation $\leq 10 \%$ was at $0.013 \mathrm{ng} / \mathrm{mL} .{ }^{21}$

NT-proBNP was assayed on a cobas e411 system (Roche Diagnostics, Mannheim, Germany) using the Roche NT-proBNP II electrochemiluminescent sandwich assay. The measuring range was $5-35,000 \mathrm{pg} / \mathrm{ml}$. The functional assay sensitivity that is the lowest analytic concentration that can be reproducibly measured with an intermediate precision CV of $20 \%$ was assessed as being $50 \mathrm{pg} / \mathrm{mL}$.

MR-proADM, MR-proANP and CT-proET-1 were measured with time resolved amplified cryptate emission (TRACE) technology on the KRYPTOR compact PLUS System (Thermo Scientific B.R.A.H.M.S GmbH, Hennigsdorf, Germany) in potassium EDTA plasma samples. The measurement range with automatic dilution for MR-proADM was 0.05 to $100 \mathrm{nmol} / \mathrm{L}$. The functional assay sensitivity (inter-assay precision of $20 \% \mathrm{CV}$ ) was assessed as being $0.25 \mathrm{nmol} / \mathrm{L}$. The measurement range with automatic dilution for MR-proANP was 2.1 to 10000 $\mathrm{pmol} / \mathrm{L}$. The functional assay sensitivity (inter-assay precision of $<20 \% \mathrm{CV}$ ) was assessed as being $10 \mathrm{pmol} / \mathrm{L}$. CT-proET-1 was measured with a research kit (Thermo Scientific B.R.A.H.M.S GmbH, Hennigsdorf, Germany).

Copeptin was measured using the Copeptin-us assay (Thermo Scientific B.R.A.H.M.S GmbH, Hennigsdorf, Germany) in potassium EDTA plasma samples with the time resolved amplified cryptate emission (TRACE) technology on a KRYPTOR compact PLUS system (Thermo Scientific B.R.A.H.M.S GmbH, Hennigsdorf, Germany). ${ }^{22}$ The limit of detection was $0.9 \mathrm{pmol} / \mathrm{L}$ and the measuring range with automatic dilution was from 0.9 to $2000 \mathrm{pmol} / \mathrm{L}$. The assay has a functional assay sensitivity (lowest value with an interassay $\mathrm{CV}<20 \%)<2.0 \mathrm{pmol} / \mathrm{L}$.

Cystatin $\mathrm{C}$, a more precise biomarker of the glomerular filtration rate, was measured using a Dako Cystatin C assay (Dako A/S, Glostrup, Denmark) in potassium EDTA plasma samples by turbidimetry on an AU 640 CC system (Beckman Coulter, Krefeld, Germany). The limit of detection was $0.028 \mathrm{mg} / \mathrm{L}$ and the measuring range 0.4 to $8.0 \mathrm{mg} / \mathrm{L}$. The 95 th percentile cut-off for individuals $>50$ years was $1.44 \mathrm{mg} / \mathrm{L}$, which was used as the diagnostic cut-off.

\section{Statistical analysis}

Continuous variables are expressed as mean \pm standard deviation, or as median ( $25 \% / 75 \%$ quartiles) for skewed variables, and categorical data as absolute numbers and 


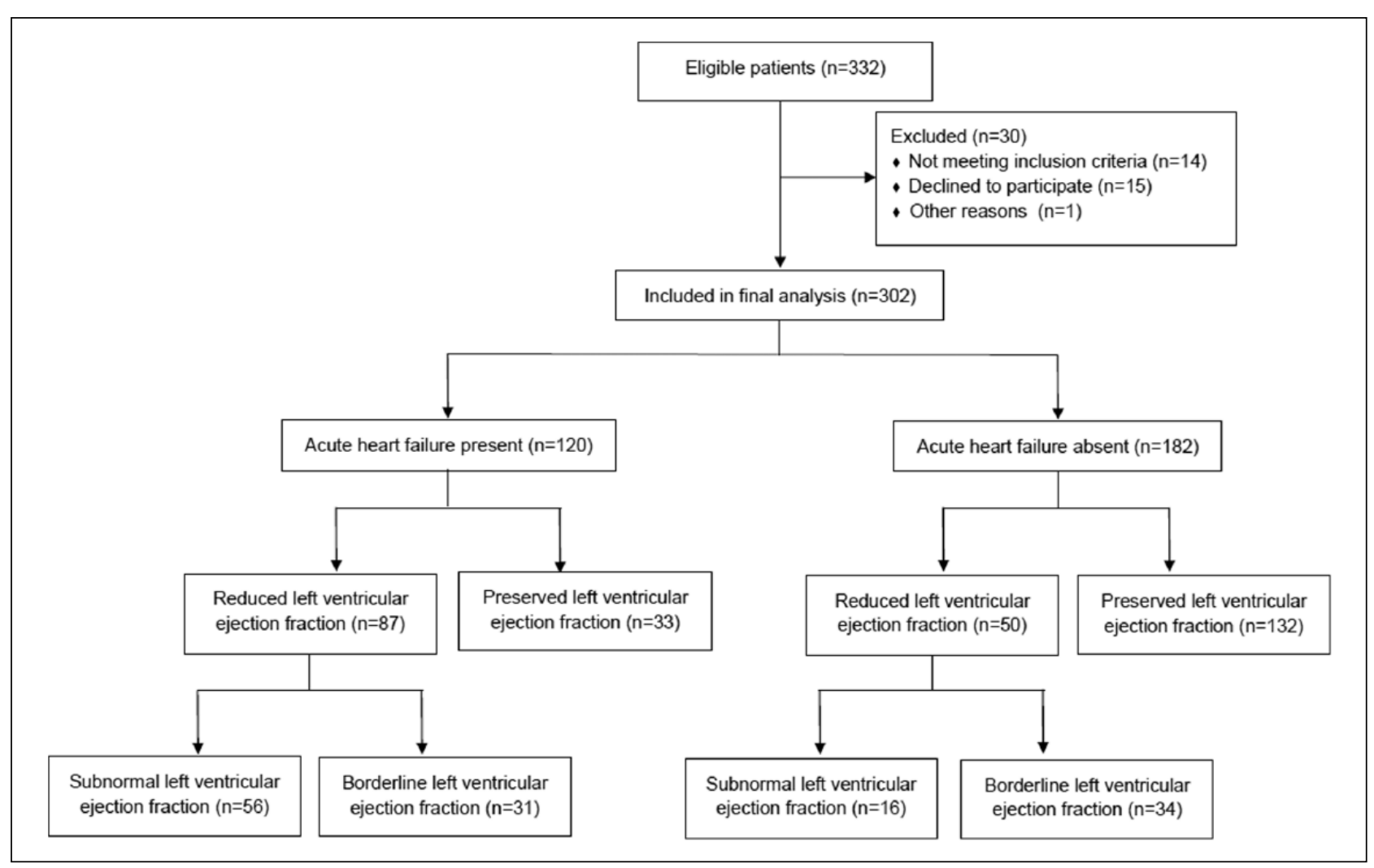

Figure I. STARD flow diagram demonstrating inclusions and exclusions according to the Standards of Reporting for Diagnostic Accuracy Studies (STARD). ${ }^{31}$

percentages. Categorical variables were compared using the Pearson Chi-Square test, while Student's t-test and analysis of variance or Mann-Whitney $U$ test were used to compare continuous variables. Receiver operating characteristics (ROC) curves were constructed to assess the sensitivity and specificity of NT-proBNP, MR-proADM, MR-proANP, CT-proET-1 and Copeptin-us assays, respectively, and to compare their ability to diagnose AHF. The comparison of areas under the ROC curve (AUC) which is equivalent to the $\mathrm{C}$-indices was performed as recommended by DeLong et al. ${ }^{23}$

The increased discriminative value of the biomarkers for diagnosis of AHF was assessed by net reclassification improvement (NRI), the continuous NRI and the integrated discrimination improvement (IDI) as described by Pencina et al. ${ }^{15}$ This metric determines the difference in the probability of a patient belonging to pre-defined risk categories before and after the addition of a specific biomarker. Categorization $(<5 \%, 5 \%-10 \%$, $10 \%-20 \%$, and $>20 \%$ ) used for the computation of NRI and continuous NRI was based on Butler et al. ${ }^{24}$ Reclassification analysis was performed using the investigated biomarkers as continuous variables in a model for the prediction of the reference standard. All models were adjusted for potential confounders such as age and gender. We evaluated reclassification comparing three incremental models: model with NT-proBNP alone (model 1), models with NT-proBNP and one additional biomarker (model 2, compared with model 1), and model with NT-proBNP and two additional biomarkers (model 3 , compared with model 2). Bootstrap confidence intervals were computed based on $B=100$ bootstrap replicates to adjust for overly optimistic results due to overfitting.

All performed tests were two-sided and a significance level of $P<0.05$ was considered. All calculations were done with $\mathrm{R}^{25}$ and IBM SPSS Statistics Version 21.0.0 (IBM Inc., Somers, NY, USA). Evaluation of diagnostic models was performed using the R package DAIM. ${ }^{26}$

\section{Results}

\section{Clinical characteristics}

We recruited 332 patients who met the inclusion criteria, of whom 30 had one or more of the prespecified exclusion criteria (Figure 1). The final population consists of 302 patients with a wide range of cardiovascular and non-cardiovascular comorbid conditions. In this cohort, the reference standard and the index tests NT-proBNP, MR-proADM, MR-proANP, CT-proET-1 and Copeptin-us were analyzed. 


\section{Adjudicated final diagnosis using the reference standard}

A final diagnosis of AHF was found in 120 patients (40\%). The baseline characteristics of patients with confirmed AHF $(n=120)$ compared to patients without AHF $(n=182)$ were different in respect to some demographic, echocardiographic and laboratory data (see Table 1 and Table 2). These patients were admitted to the ED for a variety of reasons such as chest pain, breathlessness, palpitations, lower extremity edema, functional impairment, cognitive impairment and mobility disorders. All patients were hospitalized at the index visit. The cause of HF according to the ESC classification was decompensated chronic HF in $40(33 \%)$, lung edema in $4(3 \%)$, hypertensive HF in $39(33 \%)$, cardiogenic shock in $2(2 \%)$, isolated right ventricular HF in $11(9 \%)$, and acute coronary syndrome and HF in $24(20 \%)$ patients. Most frequent comorbid conditions were a history of heart failure in $107(59 \%)$ patients, COPD in $51(28 \%)$ patients and a history of myocardial infarction in $49(27 \%)$ patients without AHF. In patients with AHF, the most frequent comorbid conditions were a history of heart failure in $116(97 \%)$ patients, diabetes with end organ damage in 56 $(47 \%)$ patients and a history of myocardial infarction in 54 $(45 \%)$ patients. Comorbid conditions as reported by the Charlson co-morbidity index were different among patients with or without AHF ( $5 \pm 3$ vs. $3 \pm 2$, respectively; $p<0.001$; see Table 1). The main findings of this study were not changed by a secondary analysis using the Charlson comorbidity index as covariate (data not shown).

\section{Multimarker approach}

Using ROC curve analysis in this population, the diagnostic accuracy with MR-proADM and NT-proBNP for AHF, quantified by the areas under the ROC curves (AUC), was significantly higher compared to NT-proBNP (AUC for MR-proADM and NT-proBNP: 0.8356 vs. AUC for NT-proBNP: $0.8103 ; p=0.045$, for comparisons; Figure 2A, 2B). The dual biomarker approach with MR-proADM and NT-proBNP yielded IDI of $3.3 \%$, NRI of $17.0 \%$ and continuous NRI of $33.3 \%$ (Table 3). Notably, the diagnostic accuracy with CT-proET-1 and NT-proBNP for AHF was significantly higher compared to NT-proBNP (AUC for CT-proET-1 and NT-proBNP: 0.8597 vs. AUC for NT-proBNP: $0.8103 ; p=0.031$, for comparisons; Figure 2A, $2 \mathrm{C})$. The dual biomarker approach with CT-proET-1 and NT-proBNP yielded robust IDI of $12.4 \%$, NRI of $40.9 \%$ and continuous NRI of $69.9 \%$ (Table 3). The triple biomarker approach MR-proADM, CT-proET-1, and NT-proBNP did not significantly improve the AUC compared to the dual biomarker strategies (Table 3; Figure 2B, 2C, 2D). Neither dual nor triple biomarker combinations with the remaining biomarkers MR-proANP or Copeptin-us significantly improved diagnosis (data not shown). When AHF patients with HFrEF or HFpEF only were investigated, none of the dual or triple biomarker combinations significantly improved diagnosis of AHF compared to NT-proBNP alone (data not shown).

\section{Discussion}

This prospective diagnostic study on AHF examined whether a multimarker strategy including MR-proADM, MR-proANP, CT-proET-1, and Copeptin-us may improve diagnostic accuracy compared to NT-proBNP alone. The main results of the study are as follows: (1) Adding MR-ADM to NT-proBNP levels significantly improved diagnosis of AHF in older patients. (2) Adding CT-proET-1 to NT-proBNP levels also significantly improved diagnostic accuracy of AHF in older patients. (3) Both dual biomarker approaches offered significant risk reclassification over NT-proBNP. (4) A triple biomarker compared to a dual biomarker approach did not further improve diagnostic accuracy.

\section{Acute heart failure in the elderly}

The prevalence of AHF in our patient cohort (40\%) was comparable to findings of recent studies. Hancock et al. found a prevalence of AHF in almost a quarter of older residents in long-term care facilities, while Oudejans et al. reported a prevalence of $46 \%$ in older patients admitted to hospital with HF symptoms. ${ }^{1,27}$ The mean age and gender distributions of patients of these studies were comparable to those of our study population. As in our study, the final diagnosis of AHF was adjudicated in those studies by two specialists independently reviewing echocardiography, ECG, and physical findings indicating high quality of analysis. Of note, the Heart Failure in Care Homes (HFinCH) Diagnostic Accuracy Study suggested that up to half of older HF patients were undiagnosed, while three-quarters of patients previously diagnosed with HF had been misclassified. ${ }^{1}$ Symptoms and signs might be substantially less clear in older patients compared to younger individuals, and they may be more difficult to detect due to the presence of one or several comorbid conditions. ${ }^{27}$

\section{Implication of study findings}

Because symptoms and signs have limited diagnostic value in older patients with suspected HF, confirmation of the diagnosis requires objective evidence of structural of functional cardiac dysfunction including echocardiography and measurement of natriuretic peptides, such as NT-proBNP. ${ }^{27-29}$ NT-proBNP is well validated and represents an acknowledged standard for diagnosis of AHF. ${ }^{4}$ While a very low concentration of NT-proBNP accurately excludes AHF, optimal cut-off levels for identifying AHF in older patients have not been clearly defined. ${ }^{13}$ Combining multiple biomarker in older patients offers 
Table I. Baseline characteristics.

\begin{tabular}{|c|c|c|c|c|}
\hline Parameter & $\begin{array}{l}\text { All patients } \\
(n=302)\end{array}$ & $\begin{array}{l}\text { Patients } \\
\text { without AHF } \\
(n=182)\end{array}$ & $\begin{array}{l}\text { Patients } \\
\text { with AHF } \\
(n=120)\end{array}$ & $p$ value \\
\hline Age, years & $80.5 \pm 5.9$ & $79.9 \pm 6.0$ & $81.4 \pm 5.8$ & 0.023 \\
\hline Male sex, $n(\%)$ & 147 (49) & $83(46)$ & $64(54)$ & 0.189 \\
\hline Body mass index, $\mathrm{kg} / \mathrm{m}^{2}$ & $27.1 \pm 5.0$ & $27.2 \pm 4.8$ & $27.1 \pm 5.2$ & 0.851 \\
\hline NYHA class & $3.0 \pm 1.0$ & $2.5 \pm 1.0$ & $3.5 \pm 0.7$ & $<0.001$ \\
\hline NYHA class I, n (\%) & $35(\mathrm{II})$ & $34(19)$ & $\mathrm{I}(\mathrm{I})$ & \\
\hline NYHA class II, n (\%) & $66(22)$ & $57(31)$ & $9(8)$ & \\
\hline NYHA class III, n (\%) & $102(34)$ & $62(34)$ & $40(33)$ & \\
\hline NYHA class IV, n (\%) & $99(33)$ & $29(16)$ & $70(58)$ & \\
\hline Barthel index & $77 \pm 25$ & $81 \pm 26$ & $73 \pm 24$ & 0.006 \\
\hline Charlson comorbidity index & $4 \pm 3$ & $3 \pm 2$ & $5 \pm 3$ & $<0.001$ \\
\hline Prescribed drugs, $n$ & $8 \pm 4$ & $7 \pm 4$ & $8 \pm 4$ & 0.017 \\
\hline Falls, $n$ & $0.8 \pm 1.4$ & $0.8 \pm 1.3$ & $0.8 \pm 1.6$ & 0.983 \\
\hline \multicolumn{5}{|l|}{ Leading symptom, $n$ (\%) } \\
\hline Angina pectoris & $72(24)$ & $46(25)$ & $26(22)$ & 0.471 \\
\hline Dyspnea & $75(25)$ & $21(12)$ & $54(45)$ & $<0.001$ \\
\hline Palpitations & $18(6)$ & $9(5)$ & $9(8)$ & 0.359 \\
\hline Malaise & $38(13)$ & $28(15)$ & $10(8)$ & 0.071 \\
\hline \multicolumn{5}{|l|}{ Cardiovascular risk factors, $n$ (\%) } \\
\hline Hypertension & $255(84)$ & $149(82)$ & $106(88)$ & 0.129 \\
\hline Hyperlipidemia & $205(68)$ & II5 (63) & $90(75)$ & 0.031 \\
\hline Diabetes mellitus & 117 (39) & $55(30)$ & $62(52)$ & $<0.001$ \\
\hline Smoking & $150(50)$ & $83(46)$ & $67(56)$ & 0.139 \\
\hline Current smoker & $20(7)$ & II (6) & $9(8)$ & 0.619 \\
\hline Former smoker & $130(43)$ & $72(40)$ & $58(48)$ & 0.132 \\
\hline Family history & $92(3 I)$ & $62(34)$ & $30(25)$ & 0.103 \\
\hline \multicolumn{5}{|l|}{ Medications, $n$ (\%) } \\
\hline Aspirin & $153(5 \mid)$ & $96(53)$ & $59(49)$ & 0.782 \\
\hline Clopidogrel & $35(12)$ & $19(10)$ & $16(13)$ & 0.475 \\
\hline ACE inhibitors & $172(56)$ & $104(57)$ & $68(57)$ & 0.718 \\
\hline Angiotensin receptor blockers & $57(19)$ & $35(19)$ & $22(18)$ & 0.715 \\
\hline Aldosterone antagonists & $10(3)$ & $7(4)$ & $3(3)$ & 0.522 \\
\hline Loop diuretics & $162(54)$ & $76(42)$ & $86(72)$ & $<0.001$ \\
\hline Thiazid diuretics & $92(30)$ & $53(29)$ & $39(33)$ & 0.563 \\
\hline Beta blockers & I7| (57) & $90(49)$ & $81(68)$ & 0.005 \\
\hline Digitalis & $36(12)$ & $13(7)$ & $23(19)$ & 0.002 \\
\hline Calcium antagonists & $95(3 \mathrm{I})$ & $54(30)$ & $41(34)$ & 0.481 \\
\hline Statins & $134(44)$ & $78(43)$ & $56(47)$ & 0.514 \\
\hline \multicolumn{5}{|l|}{ Vital status } \\
\hline Heart rate, beats per min & $85 \pm 23$ & $82 \pm 22$ & $90 \pm 25$ & 0.009 \\
\hline Respiratory rate, breaths per min & $18 \pm 4$ & $17 \pm 4$ & $20 \pm 5$ & $<0.001$ \\
\hline Systolic blood pressure, $\mathrm{mm} \mathrm{Hg}$ & $147 \pm 27$ & $150 \pm 28$ & $|4| \pm 26$ & 0.007 \\
\hline Diastolic blood pressure, $\mathrm{mmHg}$ & $75 \pm 16$ & $75 \pm 15$ & $75 \pm 17$ & 0.962 \\
\hline Oxygen saturation, \% & $95 \pm 5$ & $96 \pm 4$ & $93 \pm 6$ & $<0.001$ \\
\hline Temperature, ${ }^{\circ} \mathrm{C}$ & $36.7 \pm 0.6$ & $36.7 \pm 0.6$ & $36.7 \pm 0.6$ & 0.769 \\
\hline \multicolumn{5}{|l|}{ Echocardiography } \\
\hline Left ventricular end diastolic diameter, $\mathrm{mm}$ & $48 \pm 9$ & $46 \pm 8$ & $51 \pm 9$ & $<0.001$ \\
\hline Left ventricular end systolic diameter, $\mathrm{mm}$ & $34 \pm 10$ & $31 \pm 8$ & $39 \pm 9$ & $<0.001$ \\
\hline Left ventricular end diastolic volume, $\mathrm{ml}$ & $49 \pm 24$ & $55 \pm 26$ & $49 \pm 24$ & 0.151 \\
\hline Left ventricular end systolic volume, $\mathrm{ml}$ & $24 \pm 17$ & $30 \pm 16$ & $23 \pm 17$ & 0.026 \\
\hline Biplane left ventricular ejection fraction, $\%$ & $54 \pm 13$ & $59 \pm 10$ & $46 \pm 13$ & $<0.001$ \\
\hline Wall motion severity index & $1.4 \pm 0.5$ & $\mathrm{I} .2 \pm 0.4$ & $1.7 \pm 0.5$ & $<0.001$ \\
\hline
\end{tabular}


Table I. (Continued)

\begin{tabular}{|c|c|c|c|c|}
\hline Parameter & $\begin{array}{l}\text { All patients } \\
(n=302)\end{array}$ & $\begin{array}{l}\text { Patients } \\
\text { without AHF } \\
(n=182)\end{array}$ & $\begin{array}{l}\text { Patients } \\
\text { with AHF } \\
(n=120)\end{array}$ & $p$ value \\
\hline Left atrial end diastolic diameter, $\mathrm{ml}$ & $46.3 \pm 7.2$ & $44.5 \pm 7.0$ & $48.9 \pm 6.6$ & $<0.001$ \\
\hline Left ventricular mass index, $\mathrm{g} / \mathrm{m}^{2}$ & $208 \pm 76$ & $189 \pm 65$ & $237 \pm 82$ & $<0.001$ \\
\hline$E / e^{\prime}$ & $13.8 \pm 9.6$ & $11.9 \pm 7.6$ & $16.8 \pm 11.5$ & $<0.001$ \\
\hline$E / A$ & $1.06 \pm 0.6$ & $0.89 \pm 0.4$ & $1.45 \pm 0.8$ & $<0.001$ \\
\hline Right ventricular end diastolic diameter, $\mathrm{mm}$ & $29 \pm 7$ & $27 \pm 6$ & $31 \pm 7$ & $<0.001$ \\
\hline $\begin{array}{l}\text { Right ventricular end systolic diameter, } \mathrm{mm} \\
\text { Tricuspid annular plane systolic excursion }\end{array}$ & $\begin{array}{l}20 \pm 6 \\
2.1 \pm 0.6\end{array}$ & $\begin{array}{l}19 \pm 6 \\
2.3 \pm 0.6\end{array}$ & $\begin{array}{l}22 \pm 6 \\
1.8 \pm 0.6\end{array}$ & $\begin{array}{l}<0.001 \\
<0.001\end{array}$ \\
\hline
\end{tabular}

Data presented as $n(\%)$ of patients, mean $\pm S D$ for even variables, or median and $25 \% / 75 \%$ quartiles for skewed variables. ACE $=$ angiotensin converting enzyme, AHF = acute heart failure; NYHA = New York Heart Association. All $p$ values are descriptive only. No formal testing is considered. $P$ values describe differences between patients with or without AHF.

Table 2. Laboratory assessments.

\begin{tabular}{|c|c|c|c|c|}
\hline Parameter & $\begin{array}{l}\text { All patients } \\
(n=302)\end{array}$ & $\begin{array}{l}\text { Patients without } \\
\text { heart failure } \\
(n=182)\end{array}$ & $\begin{array}{l}\text { Patients with } \\
\text { heart failure } \\
(n=120)\end{array}$ & $p$ value \\
\hline NT-proBNP, pg/ml & $\begin{array}{l}1603 \\
(433 / 4984)\end{array}$ & $\begin{array}{l}589 \\
(303 / 1833)\end{array}$ & $\begin{array}{l}4977 \\
(2307 / 9933)\end{array}$ & $<0.001$ \\
\hline CT-proET-I, pmol/L & $\begin{array}{l}109 \\
(75 / 172)\end{array}$ & $\begin{array}{l}84 \\
(66 / / 12)\end{array}$ & $\begin{array}{l}178 \\
(125 / 240)\end{array}$ & $<0.001$ \\
\hline MR-proADM, nmol/L & $\begin{array}{l}\mid .2 \text { I } \\
(0.86 / 2.01)\end{array}$ & $\begin{array}{l}0.95 \\
(0.76 / I .3 \mid)\end{array}$ & $\begin{array}{l}1.86 \\
(1.30 / 2.94)\end{array}$ & $<0.001$ \\
\hline MR-proANP, pmol/L & $\begin{array}{l}277 \\
(166 / 47 I)\end{array}$ & $\begin{array}{l}192 \\
(141 / 293)\end{array}$ & $\begin{array}{l}464 \\
(305 / 636)\end{array}$ & $<0.001$ \\
\hline us-Copeptin, pmol/L & $\begin{array}{l}24 \\
(9 / 72)\end{array}$ & $\begin{array}{l}14 \\
(7 / 56)\end{array}$ & $\begin{array}{l}43 \\
(18 / 89)\end{array}$ & $<0.001$ \\
\hline hs-cTnT, ng/L & $\begin{array}{l}20 \\
(9 / 46)\end{array}$ & $\begin{array}{l}13 \\
(7 / 26)\end{array}$ & $\begin{array}{l}40 \\
(23 / 62)\end{array}$ & $<0.001$ \\
\hline Serum sodium, mmol/l & $\begin{array}{l}136 \\
(133 / 138)\end{array}$ & $\begin{array}{l}136 \\
(134 / 138)\end{array}$ & $\begin{array}{l}136 \\
(133 / 139)\end{array}$ & 0.147 \\
\hline Serum potassium, $\mathrm{mmol} / \mathrm{l}$ & $\begin{array}{l}4.1 \\
(3.7 / 4.4)\end{array}$ & $\begin{array}{l}4.0 \\
(3.8 / 4.4)\end{array}$ & $\begin{array}{l}4.1 \\
(3.7 / 4.5)\end{array}$ & 0.081 \\
\hline Serum creatinine, $\mathrm{mg} / \mathrm{dl}$ & $\begin{array}{l}\text { I.I } \\
(0.9 / 1.6)\end{array}$ & $\begin{array}{l}1.0 \\
(0.8 / 1.2)\end{array}$ & $\begin{array}{l}1.4 \\
(1.0 / 2.0)\end{array}$ & $<0.001$ \\
\hline Blood urea nitrogen, $\mathrm{mg} / \mathrm{dl}$ & $\begin{array}{l}47 \\
(36 / 75)\end{array}$ & $\begin{array}{l}42 \\
(33 / 53)\end{array}$ & $\begin{array}{l}63 \\
(45 / 97)\end{array}$ & $<0.001$ \\
\hline Cystatin C, mg/l & $\begin{array}{l}1.3 \\
(1.0 / 2.0)\end{array}$ & $\begin{array}{l}1.2 \\
(1.0 / 1.6)\end{array}$ & $\begin{array}{l}1.8 \\
(1.2 / 2.2)\end{array}$ & $<0.001$ \\
\hline Hemoglobin, g/dl & $\begin{array}{l}12.7 \\
(11.1 / 13.8)\end{array}$ & $\begin{array}{l}13.0 \\
(11.8 / 14.1)\end{array}$ & $\begin{array}{l}11.9 \\
(10.0 / 13.4)\end{array}$ & $<0.001$ \\
\hline Leukocytes, $10^{3} / \mu \mathrm{l}$ & $\begin{array}{l}8.3 \\
(6.3 / 10.5)\end{array}$ & $\begin{array}{l}7.8 \\
(6.2 / 10.4)\end{array}$ & $\begin{array}{l}8.8 \\
(6.9 / 10.5)\end{array}$ & 0.648 \\
\hline C-reactive protein, $\mathrm{mg} / \mathrm{dl}$ & $\begin{array}{l}0.9 \\
(0.5 / 3.2)\end{array}$ & $\begin{array}{l}0.7 \\
(0.5 / 3.0)\end{array}$ & $\begin{array}{l}1.5 \\
(0.6 / 3.4)\end{array}$ & 0.315 \\
\hline
\end{tabular}

Data presented as $n(\%)$ of patients, mean \pm SD for even variables, or median and $25 \% / 75 \%$ quartiles for skewed variables. NT-proBNP = N-terminal pro-B-type natriuretic peptide, $\mathrm{MR}$-proADM = mid-regional pro-adrenomedullin, $\mathrm{MR}$-proANP = mid-regional pro-atrial natriuretic peptide, $\mathrm{CT}$ proET-I = C-terminal pro-endothelin-I, Copeptin-us = ultra-sensitive C-terminal pro-vasopressin, hs-cTnT = high-sensitivity Troponin T. All $p$ values are descriptive only. No formal testing is considered. $P$ values describe differences between patients with or without $H F$. 
A

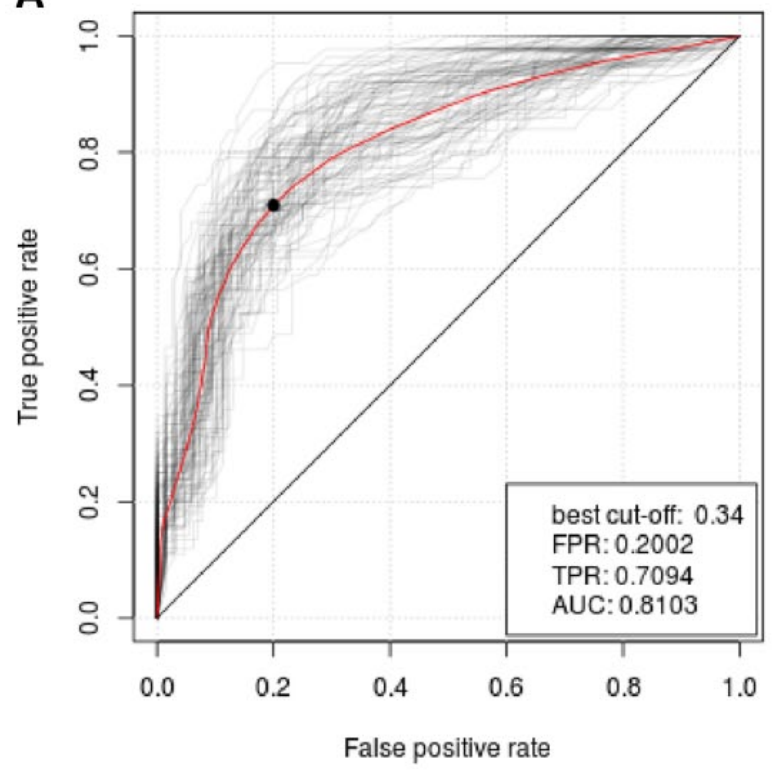

C

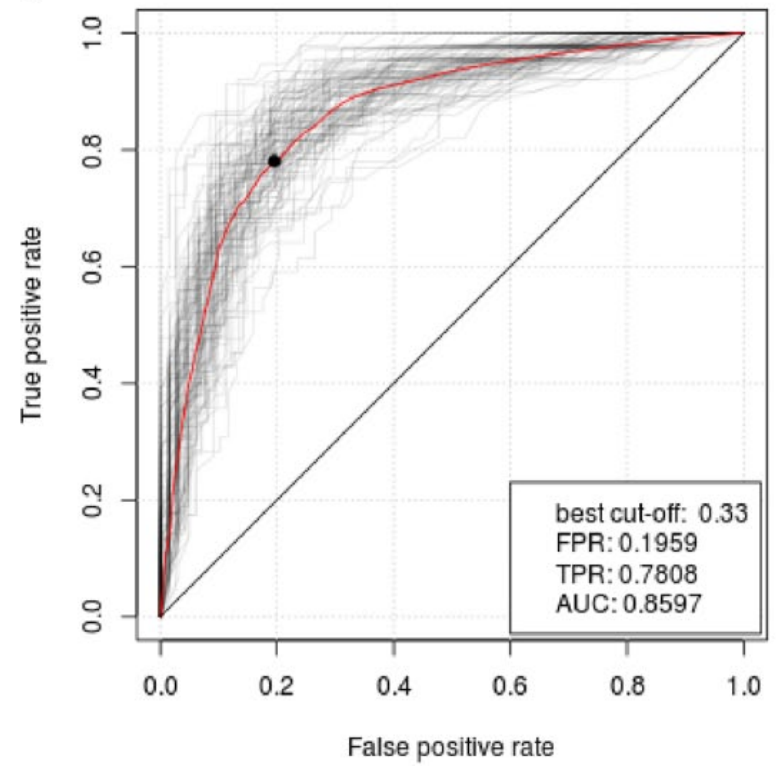

B

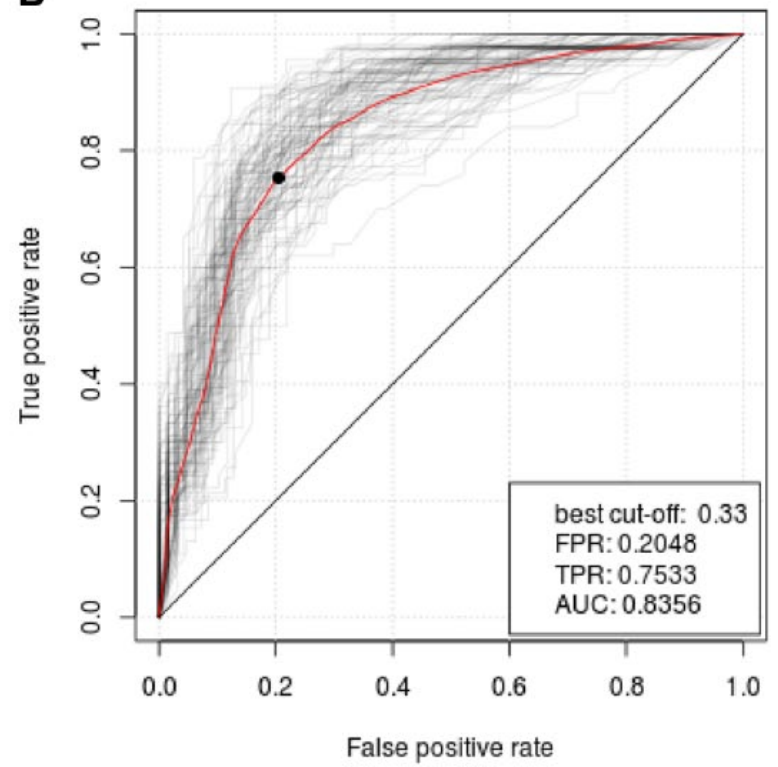

D

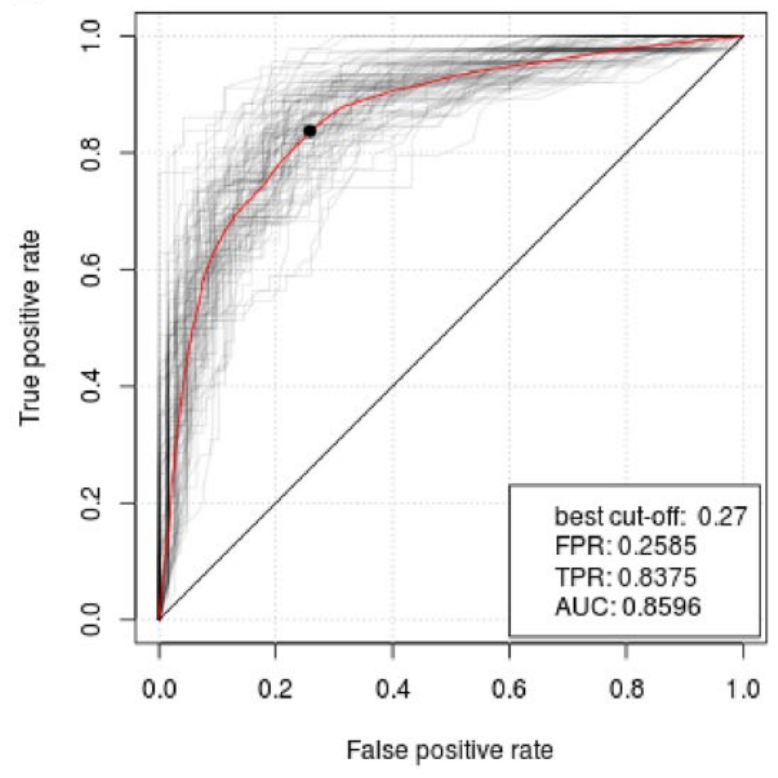

Figure 2. Receiver-operating characteristic (ROC) curves demonstrating diagnostic accuracy for AHF. All following models contain age and sex as covariates. Note that no interaction effects with either age or sex are considered. Only additive effects are considered. ROC showing the diagnostic performance for the diagnosis of AHF provided by NT-proBNP (A), NT-proBNP and MR-proADM (B), NT-proBNP and CT-proET-I (C), and NT-proBNP, CT-proET-I and MR-proADM (D). FPR indicates the false positive rate, TPR the true positive rate and $A \cup C$ the area under the curve. Grey curves represent ROC curves based on bootstrap samples, the red curve indicates the aggregated estimate over all bootstrap estimates. AHF = acute heart failure.

the potential to overcome this pitfall. Biomarkers that reflect pathophysiological pathways independent from natriuretic peptides might be more likely to provide incremental clinical benefit than biomarkers which have the same general pathophysiological phenomenon. ${ }^{2}$ In this study, NT-proBNP as a biomarker of cardiac dysfunction combined with the potent vasoconstrictor
CT-proET-1 or vasodilator MR-proADM proved to be optimal combinations of biomarkers for diagnosis of AHF. Poppe et al. demonstrated that the combination of clinical variables with a neurohormonal profile including NT-proBNP, adrenomedullin and endothelin help support identification of structural heart disease before the development of symptomatic HF. ${ }^{30}$ 
Table 3. C-statistic (AUC), IDI, NRI, and continuous NRI for progressively more complex diagnostic models of AHF adjusted for age and sex in all patients $(N=302)$. Siginicant values are highlighted in bold face.

\begin{tabular}{|c|c|c|c|c|c|}
\hline Model & \multirow{2}{*}{$\begin{array}{l}\text { Compared } \\
\text { with model no. }\end{array}$} & \multirow{2}{*}{$\begin{array}{l}\begin{array}{l}\text { AUC } \\
(p \text { value })\end{array} \\
0.8103\end{array}$} & \multirow{2}{*}{$\begin{array}{l}\text { IDI } \\
\text { ( } p \text { value })\end{array}$} & \multirow{2}{*}{$\begin{array}{l}\text { NRIa } \\
\text { ( } p \text { value })\end{array}$} & \multirow{2}{*}{$\begin{array}{l}\text { continuous } \\
\text { NRI ( } p \text { value) }\end{array}$} \\
\hline I. NT-proBNP & & & & & \\
\hline \multirow[t]{2}{*}{ 2. NT-proBNP + CT-proET-I } & 1 & 0.8597 & 0.124 & 0.409 & 0.699 \\
\hline & & $(0.03 \mathrm{I})$ & $(<0.001)$ & $(<0.001)$ & $(<0.00 \mathrm{I})$ \\
\hline \multirow{2}{*}{$\begin{array}{l}\text { 3. NT-proBNP + C } \\
+ \text { MR-proADM }\end{array}$} & 2 & 0.8596 & 0.021 & 0.077 & 0.642 \\
\hline & & $(0.4 \mid 4)$ & $(0.009)$ & $(0.006)$ & $(<0.00 \mathrm{I})$ \\
\hline \multirow[t]{2}{*}{ 4. NT-proBNP + MR-proADM } & 1 & 0.8356 & 0.033 & 0.170 & 0.333 \\
\hline & & $(0.045)$ & $(0.002)$ & $(<0.001)$ & $(0.002)$ \\
\hline $\begin{array}{l}\text { 5. NT-proBNP + MR-proADM } \\
+ \text { CT-proET-I }\end{array}$ & 4 & $\begin{array}{l}0.8596 \\
(0.198)\end{array}$ & $\begin{array}{r}0.112 \\
(<0.001)\end{array}$ & $\begin{array}{r}0.313 \\
(<0.001)\end{array}$ & $\begin{array}{c}\mathbf{0 . 7 7 1} \\
(<0.001)\end{array}$ \\
\hline
\end{tabular}

AHF = acute heart failure; NT-pro BNP = N-terminal pro B-natriuretic peptide; CT-proET-I = C-terminal pro-Endothelin-I; MR-proADM = midregional pro adrenomedullin; $A U C=$ area under the curve; IDI = integrated discrimination improvement; NRI = net reclassification improvement. aRisk of $\mathrm{AHF}$ at presentation categorized as $0 \%$ to less than $5 \%, 5 \%$ to less than $10 \%, 10 \%$ to less than $20 \%$, or $20 \%$ or greater.

While more accurate risk prediction with these dual biomarker approaches has been achieved, triple biomarker strategies failed due to significant collinearity or overlap within the multimarker panel. Our study lacked the power to perform full subgroup analysis for older patients with HFrEF or HFpEF. Mason et al. recently investigated whether the multimarker panel BNP, NT-proBNP, MR-proANP, MR-proADM, Copeptin and high-sensitivity C-reactive protein detected HFrEF or HFpEF in older people in long-term care. However no single biomarker or any combination adequately balanced rule-in and rule-out for older patients with HFrEF or HFpEF. ${ }^{31}$

Measurements of circulating biomarkers are associated with additional costs. It is a matter of concern, whether increased diagnostic costs, which are associated with improved diagnostic accuracy, is cost-effective. Of note, former work on the clinical use of natriuretic peptides on the diagnostic assessment and subsequent management of patients presenting to the ED with acute dyspnea showed that patient care is significantly improved using measurement of natriuretic peptides. In large randomized studies, it has been demonstrated that measurement of natriuretic peptides in addition to standard diagnostic care at that time, was associated with reduced hospitalization rate of affected patients, shortening of in-hospital length of stay, and a decreasing need for echocardiography or other diagnostic resources. ${ }^{32,33}$ Reported cost savings of $\$ 474$ per patient by the use of NT-proBNP measurement in addition to routine care within 60 days of follow-up. Cost savings were achieved by a reduction of hospitalizations due to rapid rule-out of AHF using NT-proBNP in the ED, and more accurate rule-in of AHF. ${ }^{32}$ Comparable data have been reported for the use of BNP. During 360 days of follow-up, $\$ 2,604$ could be saved per patient. ${ }^{33}$ Of interest, rule-in and rule-out of AHF in older patients is ambiguous and challenging due to atypical presentation of AHF in older patients and a wide variation of cut-off levels of natriuretic peptides. In addition, echocardiographic imaging is timeconsuming and challenging in older patients. Thus, it is tempting to speculate that the use of a multimarker approach may reduce overall management costs of older patients with acute dyspnea.

\section{Study limitations}

Some limitations of this diagnostic study have to be acknowledged: this study has only a moderate sample size of older patients admitted to the ED for various symptoms. Thus, a generalization of the study results should be done with caution. A validation of the current findings in larger sample size of older patients might be valuable. A definite 'gold standard' for the diagnosis of AHF is lacking and needs consideration of several features including signs and symptoms of HF including evidence for cardiac dysfunction. However, a final adjudicated diagnosis was established considering currently recommended standards supporting the high quality of this analysis. We did not limit our patient cohort to individuals presenting with shortness of breath. This was done to account for the atypical presentation of older patients with acute cardiac disease.

While the C-index and IDI provide indications of whether the new predictive model predicts AHF risk more accurately than the old model, NRI and continuous NRI indicate whether the predicted risks are sufficiently different to warrant a change in clinical decision making. Up to now, there has been no clear answer, as to which values of the statistically-significant NRIs reported in Table 3 indicate a clinically meaningful change in reclassified patients. For the NRI, equal weight is given to patients who are reclassified from the intermediate category to the low category and to patients reclassified from the intermediate to the high category, despite the fact that these changes have different clinical impact. ${ }^{34}$ An interventional study in the same patient population with death as an endpoint might 
help to solve these questions. However, the categorization used for the computation of NRI and continuous NRI in our study has been validated in large data sets for older patients with AHF. 24,35

\section{Conclusions}

In summary, measurement of biomarkers such as CT-proET-1 or MR-proADM in addition to NT-proBNP significantly improved identification of AHF in consecutive older patients presenting to the ED with a variety of symptoms. Both dual biomarker approaches offered significant risk reclassification over NT-proBNP alone, while a triple marker strategy resulted in no additional advantage.

\section{Acknowledgements}

Routine analysis and sample management were performed by Ruth Bach, Elke Pröbster and their laboratory team. MR-proADM, MR-proANP, CT-proET-1, Copeptin-us and Cystatin C were measured by Christine Aschenneller. Their highly skilled work is gratefully acknowledged.

\section{Conflict of interests}

None declared.

\section{Funding}

P.B. and A.B. are research fellows of the Robert Bosch Foundation, Stuttgart, Germany. The Robert Bosch Foundation funded this study but played no role in the design; in the collection, analysis and interpretation of data; in the writing of the report; or in the decision to submit the report for publication.

\section{References}

1. Hancock H, Close H, Mason J, et al. High prevalence of undetected heart failure in long-term care residents: findings from the Heart Failure in Care Homes (HFinCH) study. Eur $J$ Heart Fail 2013; 15: 158-165.

2. Manzano L, Escobar C, Cleland JGF, et al. Diagnosis of elderly patients with heart failure. Eur J Heart Fail 2012; 14: 1097-1103.

3. Denvir MA and Leslie SJ. Best practice for chronic heart failure patients - writing guidelines is not enough. Eur Heart J 2008; 29: 1706-1708.

4. McMurray JJ, Adamopoulus S, Anker SD, et al. ESC Guidelines for the diagnosis and treatment of acute and chronic heart failure 2012. Eur Heart J 2012; 33: 1787-1847.

5. Yancy CW, Jessup M, Bozkurt B, et al. 2013 ACCF/AHA Guideline for the Management of Heart Failure. Circulation 2013; 128: e240-e327.

6. Khunti K. Systematic review of open access echocardiography for primary care. Eur J Heart Fail 2004; 6: 79-83.

7. Fabbian F, De Giorgi A, Portaluppi F, et al. Relationship between N-Terminal Pro-B-type natriuretic peptide plasma levels and renal function evaluated with different formulae in older adult subjects admitted because of dyspnea. Gerontology 2012; 58: 50-55.
8. Mastandrea P. The diagnostic utility of brain natriuretic peptide in heart failure patients presenting with acute dyspnea: a meta-analysis. Clin Chem Lab Med 2013; 51: 1155-1165.

9. Rademaker M, Cameron V, Charles C, et al. Adrenomedullin and heart failure. Regul Pept 2003; 112: 51-60.

10. Yandle $T$ and Troughton RW. Improving risk stratification in heart failure: a role for new biomarkers? Eur J Heart Fail 2010; 12: 315-318.

11. Morgenthaler NG, Struck J, Alonso C, et al. Measurement of midregional proadrenomedullin in plasma with an immunoluminometric assay. Clin Chem 2005; 51: 1823-1829.

12. Morgenthaler NG, Struck J, Thomas B, et al. Immunoluminometric assay for the midregion of pro-atrial natriuretic peptide in human plasma. Clin Chem 2004; 50: 234-236.

13. Maisel A, Mueller C, Nowak R, et al. Mid-region prohormone markers for diagnosis and prognosis in acute dyspnea: results from the BACH (Biomarkers in Acute Heart Failure) trial. JACC 2010; 55: 2062-2076.

14. Pencina MJ, D'Agostino RB and Vasan RS. Evaluating the added predictive ability of a new marker: from area under the ROC curve to reclassification and beyond. Stat Med 2008; 27: $157-172$.

15. Pencina MJ, Steyerberg EW and D'Agostino Sr. RB. Extensions of net reclassification improvement calculations to measure usefulness of new biomarkers. Stat Med 2011; 30: $11-21$.

16. Mahoney F and Barthel D. Functional evaluation: the Barthel index. Md State Med J 1965; 14: 61-65.

17. Charlson M, Pompei P, Ales K, et al. A new method of classifying prognostic comorbidity in longitudinal studies: development and validation. J Chronic Dis 1987; 40: 373-383.

18. The Criteria Committee of the New York Heart Association. Nomenclature and criteria for diagnosis of diseases of the heart and great Vessels. Boston, MA: Little, Brown \& Co. 1994.

19. Schiller N, Shah P, Crawford M, et al. Recommendations for quantitation of the left ventricle by two-dimensional echocardiography. American Society of Echocardiography Committee on Standards, Subcommittee on Quantitation of Two-Dimensional Echocardiograms. J Am Soc Echocardiogr 1989; 2: 358-367.

20. Paulus WJ, Tschöpe C, Sanderson JE, et al. How to diagnose diastolic heart failure: a consensus statement on the diagnosis of heart failure with normal left ventricular ejection fraction by the Heart Failure and Echocardiography Associations of the European Society of Cardiology. Eur Heart J 2007; 28: 2539-2550.

21. Saenger A, Beyrau R, Braun S, et al. Multicenter analytical evaluation of a high-sensitivity troponin $\mathrm{T}$ assay. Clin Chim Acta 2011; 412: 748-754.

22. Morgenthaler NG, Struck J, Alonso C, et al. Assay for the measurement of copeptin, a stable peptide derived from the precursor of vasopressin. Clin Chem 2006; 52: 112-119.

23. DeLong E, DeLong D and Clarke-Pearson D. Comparing the areas under two or more correlated receiver operating characteristic curves: a nonparametric approach. Biometrics 1988; 44: 837-845.

24. Butler J, Kalogeropoulos A, Georgiopoulou V, et al. Incident heart failure prediction in the elderly: the Health $\mathrm{ABC}$ Heart Failure Score. Circulation: Heart Failure 2008; 1: 125-133. 
25. R Core Team. A language and environment for statistical computing. Vienna, Austria: R Foundation for Statistical Computing; 2013; Available from: http://www.R-project.org/

26. Potapov S, Adler W, Hofner B, et al. Daim: Diagnostic accuracy of classification models. R package version 1.1.0. 2013; Available from: http://CRAN.R-project.org/package=Daim

27. Oudejans I, Mosterd A, Bloemen JA, et al. Clinical evaluation of geriatric outpatients with suspected heart failure: value of symptoms, signs, and additional tests. Eur J Heart Fail 2011; 13: 518-527.

28. Kalogeropoulos A, Georgiopoulou V, DeFilippi CR, et al., for the Cardiovascular Health Study. Echocardiography, natriuretic peptides, and risk of incident heart failure in older adults. JACC: Cardiovascular Imaging 2012; 5: 131-140.

29. Wang CS, FitzGerald J, Schulzer M, et al. Does this dyspneic patient in the emergency department have congestive heart failure? JAMA 2005; 294: 1944-1956.

30. Poppe KK, Whalley GA, Richards AM, et al. Prediction of ACC/AHA Stage B heart failure by clinical and neurohormonal profiling among patients in the community. Journal of Cardiac Failure 2010; 16: 957-963.
31. Mason J, Hancock H, Close H, et al. Utility of biomarkers in the differential diagnosis of heart failure in older people: findings from the heart failure in care homes (HFinCH) diagnostic accuracy study. PLoS One 2013; 8: e53560.

32. Siebert U, Januzzi Jr JL, Beinfeld MT, et al. Costeffectiveness of using N-Terminal pro-brain natriuretic peptide to guide the diagnostic assessment and management of dyspneic patients in the emergency department. Am J Cardiol 2006; 98: 800-805.

33. Breidthardt T, Laule K, Strohmeyer AH, et al. Medical and economic long-term effects on B-Type natriuretic peptide testing in patients with acute dyspnea. Clin Chem 2007; 53: 1415-1422.

34. McGeechan K, Macaskill P, Irwig L, et al. Assessing new biomarkers and predictive models for use in clinical practice: a clinician's guide. Archives of Internal Medicine 2008; 168: 2304-2310.

35. Kalogeropoulos A, Psaty BM, Vasan RS, et al. Validation of the health abc heart failure model for incident heart failure risk prediction: the cardiovascular health study. Circulation: Heart Failure 2010; 3: 495-502. 